\title{
ANÁLISIS DEL GRADO DE IMPLANTACIÓN DE LA SOLUCIÓN TECNOLÓGICA CRM EN EL SECTOR DE LA PRODUCCIÓN DE VINOS EN ESPAÑA

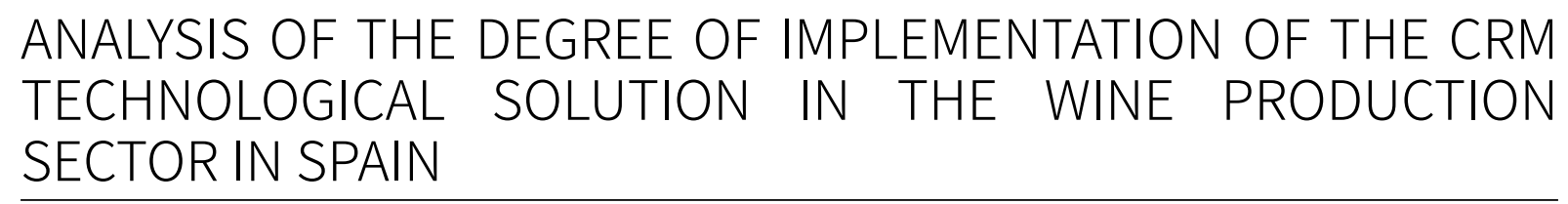

Vicente Guerola-Navarro

Departamento de Organización de Empresas. Universitat Politècnica de València, (España). E-mail: viguena@upv.es ORCID: https://orcid.org/0000-0002-0367-6319

Raul Oltra-Badenes

Departamento de Organización de Empresas. Universitat Politècnica de València, (España). E-mail: rauloltra@doe.upv.es ORCID: https://orcid.org/0000-0002-1522-8230

Hermenegildo Gil-Gomez

Departamento de Organización de Empresas. Universitat Politècnica de València, (España).

E-mail: hgil@ai2.upv.es ORCID: https://orcid.org/0000-0002-7985-2454

Andrés Sáenz-Magdalena

Departamento de Humanidades. Universidad de Navarra, (España). E-mail: asmagdalena@hotmail.com ORCID: https://orcid.org/0000-0002-8245-4326

Recepción: 09/09/2020 Aceptación: 23/11/2020 Publicación: 24/05/2021

\section{Gitación sugerida:}

Guerola-Navarro, V., Oltra-Badenes, R., Gil-Gomez, H., y Sáenz-Magdalena, A. (2021). Análisis del grado de implantación de la solución tecnológica CRM en el sector de la producción de vinos en España. 3C Empresa. Investigación y pensamiento crítico, 10(2), 17-35. https://doi.org/10.17993/3cemp.2021.100246.17-35 


\section{RESUMEN}

El sector de la producción y distribución de vinos (bodegas) es uno de los más representativos de la economía productiva española. Compuesto mayormente por pequeñas y medianas empresas, muchas de ellas familiares y poco tecnificadas, este sector afronta con dificultad el reto de la transformación digital que sus empresas necesitan para mantener su ventaja competitiva y su posición en el mercado actual. El uso, por parte de estas empresas, de algún sistema Customer Relationship Management (CRM) para la gestión de sus relaciones con los clientes, es indicativo del esfuerzo de éstas por afrontar este reto de la transformación digital.

En este este estudio se aborda, de forma científica y lejos de intereses empresariales, un completo análisis de las empresas del sector que usan algún sistema CRM, con el objetivo de detectar qué factores indican a priori una mayor predisposición a usar estos sistemas empresariales de gestión.

\section{PALABRAS CLAVE}

Transformación Digital, Tecnologías de la Información y Comunicación, Gestión de la relación con el cliente, Gestión de recursos empresariales, Bodegas, Producción y distribución de vinos. 


\section{ABSTRACT}

The wine production and distribution sector (wineries) are one of the most representative of the Spanish productive economy. Composed mostly of small and medium-sized companies, many of them family-owned and low-tech, this sector faces with diffculty the challenge of digital transformation that their companies need to maintain their competitive advantage and their position in the current market. The use, by these companies, of a Customer Relationship Management (CRM) system to manage their relationships with customers, is indicative of their efforts to face this challenge of digital transformation.

This study addresses, scientifically and far from business interests, a complete analysis of the companies in the sector that use a CRM system, in order to detect which factors, indicate a priori a greater predisposition to use these business management systems.

\section{KEYWORDS}

Digital Transformation, Information Technologies, Customer Relationship Management, Enterprise Resource Management, Wineries, Production and distribution of wines. 


\section{INTRODUCCIÓN}

La producción y distribución del vino y sus derivados es una de las actividades más representativas de la economía española. Este sector tiene por delante el reto de la transformación digital y la adopción de sistemas de gestión empresarial como los Enterprise Resource Planning (ERP) y Customer Relationship Management (CRM), que le permitan seguir siendo competitivo (Oltra-Badenes et al., 2019). La gestión de la información de mercado (a través de estos sistemas de gestión), como base de una estrategia de fidelización de clientes, serán clave en el momento económico actual caracterizado por la incertidumbre y el descenso de la demanda provocado por el confinamiento y el parón económico derivado de la pandemia del COVID-19.

Dada pues la necesidad de afrontar la transformación digital, el presente trabajo pretende averiguar cuál es la situación actual en cuanto a la implantación de sistemas de gestión (en concreto CRM, que es clave para ver cómo ha mejorado en la gestión empresarial la integración digital en la relación/satisfacción con los clientes) en el sector, como base para establecer las estrategias de adopción tecnológica que traigan una mayor eficiencia y competitividad a las empresas de este. Se trata de validar qué factores tienen relación con el grado de transformación digital y en la adopción de CRM (objetivo de nuestro enfoque de investigación) como solución de gestión por parte de las bodegas

Este estudio empieza con la definición de las características del sector vinícola y de su grado de transformación digital, junto con una revisión de la literatura referente al sector y a la tecnología CRM. Posteriormente se explica cuál ha sido el proceso de búsqueda y selección de información, y se plasman los resultados y conclusiones obtenidos.

\subsection{LA PRODUCCIÓN DE VINO Y SU DIMENSIÓN ECONÓMICA EN ESPAÑA}

España contaba con 4.373 bodegas a finales de 2018 (Tecnovino, 2018; OEMV, 2018), con tendencia claramente creciente según el Observatorio Español del Mercado del Vino (OeMv), datos proporcionados por el Directorio Central de Negocios (DIRCE) del INE. El sector de la producción de vino es uno de los 
más importantes en España, con un claro objetivo de calidad y la satisfacción del cliente (CECRV; 2020). El valor de la producción del sector del vino y el mosto, dentro de la categoría de los ingresos agrícolas, ha crecido en 2019 un 26\% respecto a 2018, llegando a 2.142,8 millones de euros (valores actuales a precios básicos), lo cual representa un 8.02\% de la producción de vegetales y casi $4.8 \%$ de la producción agrícola. El empleo dedicado al cultivo de la viña genera 18 millones de salarios (MAPA, 2020). En cuanto a superficie dedicada al cultivo de la viña, el Ministerio de Agricultura, Pesca y Alimentación (MAPA) recoge en sus datos estadísticos que, en España, y según los datos del Registro Vitivinícola de cada comunidad autónoma al 31 de julio de 2019 asciende a 957.573 hectáreas de terreno agrícola.

\subsection{TRANSFORMACIÓN DIGITAL EN EL SECTOR DE LAS BODEGAS EN ESPAÑA}

Los informes actuales certifican que la mayoría de las bodegas españolas tienen el carácter de pequeñas y medianas empresas (PYME), en muchos casos empresas familiares (MAPA, 2020; OEMV, 2018). A priori, este factor de tamaño parece que puede influir en las dificultades para enfrentar el desafío de la transformación digital y la adopción de tecnologías modernas de gestión empresarial (Ferrer-Lorenzo et al.; 2017), como lo son los ERP y CRM.

Los grandes grupos de bodegas suelen tener departamentos tecnológicos que desarrollan su propia tecnología de gestión. Por otro lado, la mayor parte del tejido empresarial de las bodegas son empresas familiares que no requieren una gran tecnología, porque cuentan con muy pocos empleados y los procesos de gestión suelen ser muy simples. Por lo general, trabajan con programas básicos de contabilidad y facturación, y algunas aplicaciones de gestión de almacenes.

\section{REVISIÓN DE LITERATURA}

\subsection{CUSTOMER RELATIONSHIP MANAGEMENT}

Las primeras referencias a la solución tecnológica CRM aparecen en la década de 1970, siendo en sus inicios una solución centrada en la automatización de la fuerza de ventas (Guerola-Navarro et al., 2020a). 
El alcance de esta solución tecnológica ha ido creciendo, siempre dentro de un enfoque estratégico de gestión centrada en el cliente, hacia dos ámbitos adicionales: el Marketing, y el soporte y atención al cliente (Servicios).

El análisis bibliométrico sobre los estudios de CRM muestra que la relevancia de CRM como una solución clave para el éxito de los tomadores de decisiones empresariales en el desarrollo de sus funciones diarias, así como dentro del campo de la investigación científica, ha estado creciendo significativamente a partir del año 2000, y ya de forma exponencial a partir del año 2010 (Guerola-Navarro et al., 2020a). Este enfoque cuantitativo de la realidad de CRM, junto con el enfoque cualitativo proporcionado por una Revisión de Literatura extendida (Guerola-Navarro et al., 2020a), representan una imagen clara de CRM como foco creciente de interés por la investigación científica, con un repositorio aún no abundante de estudios de referencia, pero con una base de datos de bibliografía en continuo crecimiento que hace de CRM uno de los pilares básicos de estudio en el campo de la búsqueda de la excelencia empresarial a través de estrategias de gestión centradas en el cliente.

Algunos estudios se han realizado ya en etapas recientes sobre los beneficios esperados de laimplementación y uso de CRM en las empresas (Gil-Gomez et al., 2020; Guerola-Navarro et al., 2020b), desde el doble punto de vista que le confiere las características tan valiosas hoy en día como son la "sostenibilidad" y "ambidexterity":

- Explotación de los beneficios actuales mediante una gestión efectiva y eficiente de los recursos en el ámbito de la gestión global e integrada de recursos empresariales

- Exploración de los beneficios futuros de una estrategia centrada en el conocimiento del cliente y en la generación de información valiosa de gestión a largo plazo para el establecimiento de relaciones de confianza duraderas con los socios comerciales de la empresa. 
Existe poca literatura publicada actualmente sobre la validación empírica de estos beneficios esperados, si bien ya hay estudios que proponen modelos de validación e investigación completos Guerola-Navarro et al. (2020c).

El objetivo del presente estudio es hacer una primera investigación sobre un sector concreto, el sector de la producción de vinos (bodegas) en España, uno de los más representativos de nuestro país. En estudios posteriores, y en base a las conclusiones de este estudio, se probará empíricamente la relación entre el grado de introducción (y uso) de CRM en estas empresas y la mejora en rendimiento obtenida en las mismas a consecuencia de la gestión empresarial realizada mediante CRM.

\subsection{CRM Y EL SECTOR DEL VINO}

Existe muy poco volumen de publicaciones de investigación científica en el ámbito del sector de la producción de los vinos, reduciéndose a un volumen realmente mínimo cuando además se añade el criterio de búsqueda referente a las Tecnologías de Información y Comunicación (TIC), la Transformación Digital, y más en concreto la adopción y uso de CRM. Solo 53 publicaciones se han registrado en la Web Of Science entre 1900 y 2020 coincidiendo los criterios de "Winery" y "Wine Production" juntamente con los de "Information Technology", "ERP" y "CRM" (con sus variantes y nombres extensivos, y entendiendo que ERP y CRM son las dos TIC más populares).

Belias et al. (2018) revisa desde un punto de vista crítico la literatura existente sobre el impacto del uso de CRM en los sectores del vino, las bodegas y el enoturismo, concluyendo que todavía hay un largo camino por recorrer antes de tener una idea clara de los beneficios que se esperan para llevar a estos sectores el uso de CRM. Las sugerencias reflejadas en los estudios existentes sobre el impacto de CRM en el rendimiento de la empresa, en conjunto con los que se refieren a la modernización del sector de las bodegas, predicen una clara expectativa positiva sobre el impacto del uso de CRM en los indicadores de rendimiento de la empresa, pero todavía no hay estudios concretos al respecto. Este estudio se centra en el sector vitivinícola turístico griego. 
Crescimanno et al. (2017) es otra investigación local relacionada con el sector vitivinícola y las TI, que revisa específicamente el impacto de las redes sociales $(\mathrm{SM})$ en el sector vitivinícola italiano. En este caso, el estudio refleja los claros efectos de SM en la imagen corporativa y la gestión de la relación con la cliente desarrollada a través de todos los departamentos y declaraciones de la empresa. En este caso, la solución tecnológica de CRM no es la protagonista, ni el foco del estudio, pero parece mostrar una forma interesante para futuras investigaciones e investigaciones.

Ferrer-Lorenzo et al. (2017) impulsa un estudio específico del sector vitivinícola español, profundizando en el impacto en el rendimiento empresarial. En este caso, se tiene en cuenta las diferencias entre grupos empresariales y empresas independientes, señalando el impacto de las diferencias de tamaño y estructura. Una de las conclusiones se refiere a la forma más compleja y consciente que utilizan las grandes empresas para impulsar estrategias de marketing centradas en el cliente, siempre a través de estrategias de gestión de relaciones con los clientes, pero no siempre a través de soluciones tecnológicas de CRM. El objetivo de los grupos empresariales mediante el uso de tecnologías modernas de gestión del conocimiento es frecuentemente compartir información común. Aunque, en este documento, no hay un modelo de medición para validar el impacto del uso de estas tecnologías.

Podemos concluir pues que el impacto del uso de CRM, y aún más en general el impacto del uso de cualquiera de las TIC más extendidas, específicamente relacionado con el sector de las bodegas, no se ha estudiado previamente. Esta conclusión da un claro valor y originalidad al presente documento, ya que tiene el objetivo final de establecer parámetros indicativos de las condiciones que hacen que CRM sea de interés para las empresas españolas dedicadas a la producción de vinos.

\section{METODOLOGÍA}

Se ha procedido inicialmente a la búsqueda de una base de datos fiable y coherente con información sobre las empresas del sector en estudio. Posteriormente se ha procedido a la selección de las empresas objetivo de estudio, junto con la información pública disponible sobre sus cifras de negocio. Una vez 
seleccionadas las empresas, y recopilada toda la información de interés, se ha clasificado los componentes determinantes en función de su relevancia dentro del conjunto de empresas en estudio.

\subsection{SELECCIÓN DE LAS EMPRESAS}

Con el objetivo de analizar qué factores aparecen comúnmente en las empresas de producción de vinos españolas que usan CRM, y así tratar de determinar cuáles de estos factores podrían llegar a tratarse como condición necesaria y/o suficiente para que CRM sea considerada una solución de gestión empresarial clave, el presente estudio ha investigado cuál es el segmento más representativo dentro de las bodegas de España.

El criterio de selección para obtener la base de datos de la empresa como objeto de estudio ha sido elegir, entre las 2.575 empresas españolas registradas en el Instituto Nacional de Estadística (INE) con el código GNAE-1 102 (correspondiente al encabezado "Producción y elaboración de vinos"), seleccionar aquellas cuya facturación es al menos de 2 millones de euros anuales. Este criterio ha sido elegido para tener un número suficiente de elementos de estudio, y que estos elementos de estudio sean representativos del sector. Con este criterio se ha incluido en el estudio, desde las diez corporaciones vitivinícolas más grandes del mercado español (con más de 10 millones de euros de facturación anual), representadas por grupos multinacionales, hasta las empresas que muestran un grado razonable de transformación digital y de adopción de tecnologías TIC actualmente. Esta base de datos se obtuvo del informe Axesor (2019), a partir del 01/10/2019 con los datos públicos del Registro Mercantil y obteniendo un total de 418 empresas por encima de 2 millones de euros de facturación anual. En un muestreo de empresas por debajo de los 2 millones de facturación anual, la ratio de adopción de TIC desciende exponencialmente, lo cual constata que el criterio elegido es el adecuado.

De estas 418 empresas, todas ellas con su nombre corporativo ubicadas en España, dedicadas a la producción de vinos (GNAE 1102), y con más de 2 millones de euros de facturación, se ha seleccionado las que han implantado y están utilizando algún sistema de CRM. El proceso de obtención de información 
sobre el uso de algún sistema CRM, se ha realizado mediante llamadas individuales a las 418 empresas. Se ha obtenido de este modo información sobre las 84 empresas (de entre las 418 iniciales) que cuentan con algún tipo de CRM en uso y en producción. Con esta información se ha procedido a clasificar y categorizar los elementos más relevantes sobre las empresas del sector de producción de vinos que usan CRM.

\subsection{CLASIFICACIÓN DE LAS EMPRESAS}

Una vez identificadas las 84 empresas que, siendo representativas del sector, cuentan con algún tipo de CRM en uso, se procede a analizar la información de que se dispone, para evaluar qué parámetros pueden ser relevantes como condiciones de adopción de GRM por parte de las bodegas.

Los parámetros elegidos como representativos de la dimensión de la actividad empresarial de cada entidad han sido:

- Rango de facturación anual.

- Rango de número de empleados.

- Localización geográfica.

Los parámetros adicionales, y representativos del grado de transformación tecnológica por parte de las bodegas, han sido la identificación de su:

- ERP.

- CRM.

Estos 5 parámetros, los tres primeros públicos y comparables, y los dos últimos proporcionados por las bodegas en las llamadas realizadas, se usan en este estudio para analizar su incidencia dentro del parque de las 84 empresas de producción y elaboración de vinos en España que usan CRM.

\section{RESULTADOS}




\subsection{CLASIFICACIÓN SEGÚN EL SISTEMA CRM EN USO}

El objetivo principal de este estudio es obtener un punto de partida en cuanto a la situación de desarrollo de la tecnología de gestión CRM dentro del sector de producción de vino español.

Las 84 empresas que han reconocido estar usando, en mayor o menor grado, CRM como solución de gestión, representan un 20,10\% del total de bodegas con una facturación anual mayor de 2 millones de euros.

La Tabla 1 analiza en detalle, de las 84 entidades que usan CRM, su distribución por fabricantes del software de gestión es la siguiente:

Tabla 1. Distribución de empresas según el CRM que usan.

\begin{tabular}{|c|c|}
\hline CRM & Porcentaje de empresas \\
\hline WOLF - CRM & $41,27 \%$ \\
\hline Dynamics NAV - CRM & $9,52 \%$ \\
\hline SAP - CRM & $9,52 \%$ \\
\hline SOLMICRO - CRM & $6,35 \%$ \\
\hline Dynamics CRM & $4,76 \%$ \\
\hline SAGE - CRM & $4,76 \%$ \\
\hline SALESFORCE & $4,76 \%$ \\
\hline EUROVIN - CRM & $3,17 \%$ \\
\hline COPERMATICA & $1,59 \%$ \\
\hline EKON - CRM & $1,59 \%$ \\
\hline EUROMUS & $1,59 \%$ \\
\hline EXPERTIS & $1,59 \%$ \\
\hline GOLDMINE & $1,59 \%$ \\
\hline ISAGRI & $1,59 \%$ \\
\hline IWINE - CRM & $1,59 \%$ \\
\hline VISUAL-CRM & $1,59 \%$ \\
\hline WINCHE & $1,59 \%$ \\
\hline ZOHO & $1,59 \%$ \\
\hline
\end{tabular}

Fuente: elaboración propia. 
Constatamos un claro liderazgo de WOLF-CRM dentro de las empresas del sector, con un 41,27\% del total. Si consideramos, ya de lejos, pero con una buena cuota de mercado al módulo CRM de Dynamics NAV $(9,52 \%)$ y al módulo CRM de SAP (9,52\%), entre las tres alcanzan una cuota de mercado del 60,32\%. Agregando al cuarto fabricante (módulo CRM de SOLMICRO, con un 6,35\%), entre los cuatro alcanzan un $66,67 \%$, que son dos tercios del mercado.

\subsection{CLASIFICACIÓN SEGÚN EL ERP EN USO}

Una vez categorizadas las empresas según el sistema CRM que usan, es interesante evaluar cuál es la distribución por uso de ERP, que es la otra tecnología de gestión empresarial más extendida en el mercado. Con ello, y juntamente con la distribución de CRM, se pretende obtener la visión actual del grado de transformación digital en el área de sistemas integrados de gestión empresarial.

De entre las 84 empresas dedicadas a la producción de vinos, y por encima de los 2 millones de facturación anual, la distribución entre ellas de los sistemas ERP en uso es la que muestra la Tabla 2.

Tabla 2. Distribución de empresas según el ERP que usan.

\begin{tabular}{|c|c|}
\hline ERP & Porcentaje de empresas \\
\hline SAP & $28,13 \%$ \\
\hline Dynamics NAV & $21,88 \%$ \\
\hline SOLMICRO & $18,75 \%$ \\
\hline EUROVIN & $9,38 \%$ \\
\hline SAGE & $6,25 \%$ \\
\hline ALFA & $3,13 \%$ \\
\hline DATAWINE & $3,13 \%$ \\
\hline ISAGRI & $3,13 \%$ \\
\hline IWINE & $3,13 \%$ \\
\hline SALESFORCE & $3,13 \%$ \\
\hline
\end{tabular}

Fuente: elaboración propia.

Se observa un claro liderazgo de los productos SAP, Dynamics NAV, y SOLMICRO, cubriendo entre las tres el $68,75 \%$ del total de las empresas. 
Interesante también es comprobar que los tres mayores representantes como fabricantes de ERP en el sector (SAP, Dynamics NAV, y SOLMICRO), coinciden con el segundo, tercero y cuarto mayor fabricante de CRM (según la Tabla 1).

\subsection{CLASIFICACIÓN SEGÚN LA FACTURACIÓN}

La Tabla 3 muestra cómo se distribuyen las 84 empresas que tienen CRM según su nivel de facturación anual, lo cual nos permite estratificar por nivel de facturación las bodegas que usan CRM.

Tabla 3. Clasificación de las empresas que usan CRM, según su facturación anual en 2018.

\begin{tabular}{|c|c|c|}
\hline FACTURACIÓN & Empresas con CRM & Porcentaje con CRM \\
\hline 2 - 10 MILLONES EUR & 55 & $65,48 \%$ \\
\hline $10-50$ MILLONES EUR & 28 & $33,33 \%$ \\
\hline MÁS DE 50 MILLONES EUR & 1 & $1,19 \%$ \\
\hline TOTAL & $\mathbf{8 4}$ & $\mathbf{1 0 0 , 0 0 \%}$ \\
\hline
\end{tabular}

Fuente: elaboración propia.

En términos absolutos, dos terceras partes de las empresas del sector que usan CRM, tienen un nivel de facturación de entre 2 y 10 millones de euros.

Por otro lado, y con el objetivo de detectar cual es el segmento de facturación con mayor nivel de penetración de GRM en términos relativos, se presenta la Tabla 4, que muestra qué porcentaje de las 418 empresas del sector tiene CRM.

Tabla 4. Grado de penetración de CRM por rangos de facturación anual en 2018.

\begin{tabular}{|c|c|c|c|}
\hline FACTURACIÓN & Total de empresas & Empresas con CRM & Porcentaje con CRM \\
\hline 2 - 10 MILLONES EUR & 330 & 55 & $16,67 \%$ \\
\hline $10-50$ MILLONES EUR & 74 & 28 & $37,84 \%$ \\
\hline MÁS DE 50 MILLONES EUR & 14 & 1 & $7,14 \%$ \\
\hline TOTAL & $\mathbf{4 1 8}$ & $\mathbf{8 4}$ & $\mathbf{2 0 , 1 0 \%}$ \\
\hline
\end{tabular}

Fuente: elaboración propia.

El rango de facturación en el que mayor porcentaje de empresas tiene CRM es el de 10 a 50 millones de euros anuales, donde algo más de una de cada tres empresas (el 37,84\%) usa CRM. 


\subsection{CLASIFICACIÓN SEGÚN EL NÚMERO DE EMPLEADOS}

Las empresas en España tienen la categoría de "Pequeñas" por debajo de 50 empleados, "Medias" entre 50 y 250 empleados, y “Grandes” por encima de 250 empleados. Siguiendo esta clasificación, la Tabla 5 muestra la cantidad absoluta de empresas que usan CRM, clasificada por tamaño de empresa.

Tabla 5. Clasificación de las empresas que usan CRM, según el número de empleados.

\begin{tabular}{|c|c|c|}
\hline EMPLEADOS & Empresas con CRM & Porcentaje con CRM \\
\hline Pequeña $(<50)$ & 63 & $75,00 \%$ \\
\hline Mediana $($ entre 50 y 250$)$ & 20 & $23,81 \%$ \\
\hline Grande $(>250)$ & 1 & $1,19 \%$ \\
\hline TOTAL & $\mathbf{8 4}$ & $\mathbf{1 0 0 , 0 0 \%}$ \\
\hline
\end{tabular}

Fuente: elaboración propia.

Con gran diferencia, un 75\% del total de empresas que usan CRM es de tamaño pequeño (menos de 50 empleados). La Tabla 6 muestra, en términos relativos, la penetración de CRM por tamaño de empresa.

Tabla 6. Grado de penetración de CRM por rangos de número de empleados.

\begin{tabular}{|c|c|c|c|}
\hline FACTURACIÓN & Total de empresas & Empresas con CRM & Porcentaje con CRM \\
\hline Pequeña $(<50)$ & 367 & 63 & $17,17 \%$ \\
\hline Mediana (entre 50 y 250) & 47 & 20 & $42,55 \%$ \\
\hline Grande $(>250)$ & 4 & 1 & $25,00 \%$ \\
\hline TOTAL & $\mathbf{4 1 8}$ & $\mathbf{8 4}$ & $\mathbf{2 0 , 1 0 \%}$ \\
\hline
\end{tabular}

Fuente: elaboración propia.

El segmento con mayor penetración es pues el de la Mediana empresa, con casi la mitad de las mismas usando CRM.

\subsection{DISTRIBUCIÓN POR ZONA GEOGRÁFICA}

El último criterio de clasificación empleado es el de la distribución geográfica. Este sector goza de particularidades muy específicas por zona geográfica, con gestión protegida a través de las Denominaciones Protegidas de Origen (DPO) y características diferenciadas del producto por cada área y DPO (CECRV, 2020). La mayor parte de las DPO van ligadas a zonas geográficas, por lo que se 
considera interesante también clasificar el número de empresas que usan CRM según la zona geográfica a la que pertenecen. La Tabla 7 muestra, de las 84 empresas que usan CRM, cómo se distribuyen por Comunidades Autónomas.

Tabla 7. Distribución geográfica de las empresas que usan CRM.

\begin{tabular}{|c|c|c|}
\hline Comunidad Autónoma & Empresas que usan CRM & Porcentaje \\
\hline CATALUÑA & 17 & $20,24 \%$ \\
\hline CASTILLA Y LEÓN & 15 & $17,86 \%$ \\
\hline PAís VASCO & 8 & $9,52 \%$ \\
\hline ANDALUCíA & 6 & $7,14 \%$ \\
\hline MADRID & 6 & $7,14 \%$ \\
\hline MURCIA & 6 & $7,14 \%$ \\
\hline ARAGÓN & 5 & $5,95 \%$ \\
\hline CASTILLA LA MANCHA & 5 & $5,95 \%$ \\
\hline COMUNIDAD VALENCIANA & 5 & $5,95 \%$ \\
\hline GALICIA & 5 & $5,95 \%$ \\
\hline LA RIOJA & 3 & $3,57 \%$ \\
\hline NAVARRA & 2 & $2,38 \%$ \\
\hline ASTURIAS & 1 & $1,19 \%$ \\
\hline TOTAL & 84 & $100,00 \%$ \\
\hline
\end{tabular}

Fuente: elaboración propia.

En términos relativos, la Tabla 8 muestra, de las empresas totales de cada Comunidad Autónoma, cuantas y qué porcentaje de las empresas de cada Autonomía usan CRM.

Tabla 8. Grado de penetración de CRM según la distribución geográfica de las empresas.

\begin{tabular}{|c|c|c|c|}
\hline Comunidad Autónoma (CCAA) & Empresas por CCAA & $\begin{array}{c}\text { Empresas por CCAA con } \\
\text { CRM }\end{array}$ & Porcentaje con CRM \\
\hline ASTURIAS & 1 & 1 & $100,00 \%$ \\
\hline PAís VASCO & 26 & 8 & $30,77 \%$ \\
\hline MURCIA & 21 & 6 & $28,57 \%$ \\
\hline CATALUÑA & 67 & 17 & $25,37 \%$ \\
\hline ARAGÓN & 20 & 5 & $25,00 \%$ \\
\hline CASTILLAY LEON & 63 & 15 & $23,81 \%$ \\
\hline
\end{tabular}




\begin{tabular}{|c|c|c|c|}
\hline MADRID & 28 & 6 & $21,43 \%$ \\
\hline GALICIA & 24 & 5 & $20,83 \%$ \\
\hline COMUNIDAD VALENCIANA & 27 & 5 & $18,52 \%$ \\
\hline ANDALUCÍA & 35 & 6 & $17,14 \%$ \\
\hline NAVARRA & 13 & 2 & $15,38 \%$ \\
\hline LA RIOJA & 21 & 3 & $14,29 \%$ \\
\hline CASTILLA LA MANCHA & 47 & 5 & $10,64 \%$ \\
\hline BALEARES & 5 & 0 & $0,00 \%$ \\
\hline CANARIAS & 6 & 0 & $0,00 \%$ \\
\hline EXNTABRIA & 1 & 0 & $0,00 \%$ \\
\hline TREMADURA & 13 & 0 & $0,00 \%$ \\
\hline
\end{tabular}

Fuente: elaboración propia.

Claramente destacada aparece Asturias con el 100\% de grado de penetración (la única empresa de la muestra localizada allí usa CRM). Comunidades como País Vasco, Murcia, Cataluña, Aragón, Castilla y León, Madrid y Galicia aparecen con entre un 20\% y 30\% de sus empresas usando CRM. El estudio también reporta que Comunidad Valenciana, Andalucía, Navarra, La Rioja y Castilla La Mancha tienen entre un $10 \%$ y un $20 \%$ de sus empresas usando CRM.

\section{CONCLUSIONES}

Las empresas que usan CRM, dentro del total de empresas analizadas en el sector, representan un $20,10 \%$ del total. Este grado de penetración es muy inferior al de otros sectores productivos, lo cual otorga un potencial muy grande de expansión y muy buenas expectativas futuras en un mercado que cada vez requiere de una mayor y mejor gestión de clientes enfocada a la gestión del conocimiento y la fidelización de clientes como estrategia exitosa de ventas, marketing, servicios y atención al cliente.

En cuanto a la clasificación por fabricantes de CRM que tienen despliegues en el sector, se ve una clara polarización, con un fabricante que cubre más del 40\% del mercado. Esto se refuerza con el hecho de que este CRM, junto con los tres siguientes, alcanzan las dos terceras partes del mercado. 
Por lo que respecta a los ERP implantados en el sector, los tres mayores representantes como fabricantes de ERP en el sector coinciden con el segundo, tercero y cuarto mayor fabricante de CRM, lo cual es de nuevo indicador de la concentración y polarización del sector de las TIC en unos pocos fabricantes. El hecho de que los productos de un mismo fabricante tengan resuelta de serie la integración entre los mismos, también ayuda a esta realidad.

Por rangos de facturación, donde más cantidad de empresas hay usando CRM es en el nivel de facturación de entre 2 y 10 millones de euros, lo cual es lógico porque es donde mayor cantidad de empresas hay. En términos relativos, el rango de facturación en el que mayor grado de penetración existe por parte de CRM es en el de 10 a 50 millones de euros (el 37,84\% de estas empresas usan CRM), seguido por las empresas entre 2 y 10 millones de euros (aquí ya solo el 16,67\% de las mismas usan CRM) y solo un 7,14\% de las de más de 50 millones de euros usan CRM.

Respecto al tamaño de la empresa, medido por el número de empleados, un 75\% del total de empresas que usan CRM es de tamaño pequeño (menos de 50 empleados). Sin embargo, en términos relativos, el segmento con mayor penetración es pues el de la Mediana empresa (entre 50 y 250 empleados) con un 42,55\% de las mismas usando CRM, seguido por las empresas Grandes (más de 250 empleados) donde el 25\% de las mismas usan CRM, y ya por debajo solo el 17,17\% de las empresas Pequeñas (menos de 50 empleados) usan CRM.

Por Comunidades Autónomas, la que más empresas tiene usando CRM es Cataluña que cuenta con el 20,24\% del total de empresas españolas, seguida por Castilla León con el 17,86\% del total. El estudio del grado de penetración por Comunidades Autónomas no reporta grandes diferencias entre Autonomías.

Con estos datos, y las conclusiones obtenidas, queda definido cual es el estado de despliegue de la solución tecnológica CRM en el sector de la producción de vinos en España. En base a esta área de trabajo, se propone posteriores estudios sobre la relación entre cada uno de los factores analizados en 
esta investigación, así como la determinación de la necesidad y/o suficiencia de cada uno de ellos como condición para que las empresas del sector usen CRM.

\section{REFERENCIAS BIBLIOGRÁFICAS}

Axesor. (2019). Informe de facturación de empresas con CNAE 1102. https://www.axesor.es/

Belias, D., Velissariou, E., Kyriakou, D., Vasiliadis, L., Mantas, G., Sdrolias, L., Aspridis, G., y Kakkos, N. (2018). The importance of customer relationship management and social media in the Greek wine tourism industry. In Innovative approaches to tourism and leisure (pp. 249-259). Springer, Cham. https://ideas.repec.org/h/spr/prbchp/978-3-319-67603-6_19.html

GEGRV (Conferencia Española de Consejos Reguladores Vitivinícolas). (2020). El origen de las DDOO. https://vinosdo.wine/denominaciones-de-origen/origen/

Grescimanno, M., Galati, A., Tulone, A., y Tinervia, S. (2017). Social media technology use and managers perception. A preliminary study in the italian wine industry. In 10th Annual Conference of the EuroMed Academy of Business, (pp. 474-485). https://emrbi.org/wp-content/uploads/2020/09/ EuroMed-10-2017.pdf

Ferrer-Lorenzo, J. R., Abella-Garcés, S., y Maza-Rubio, T. (2017). Competitive advantage differences between firms belonging to a business group and independent companies in the Spanish wine industry. Economía Agraria y Recursos Naturales-Agricultural and Resource Economics, 17(2), 105-132. https://doi.org/10.7201/earn.2017.02.05

Gil-Gomez, H., Guerola-Navarro, V., Oltra-Badenes, R., y Lozano-Quilis, J. A. (2020). Customer relationship management: digital transformation and sustainable business model innovation. Economic Research-Ekonomska Istraživanja, 33(1), 2733-2750. https://doi. org/10.1080/1331677X.2019.1676283 
Guerola-Navarro, V., Oltra-Badenes, R., Gil-Gomez, H., y Gil-Gomez, J. A. (2020a). Customer relationship management (CRM): a bibliometric analysis. International fournal of Services Operations and Informatics, 10(3), 242-268. https://doi.org/10.1504/IJSOI.2020.108988

Guerola-Navarro, V., Oltra-Badenes, R., y Gil-Gómez, H. (2020b). Análisis de la relación entre el grado de introducción de CRM y los beneficios de la empresa a través del desempeño organizacional y la innovación empresarial. 3C Empresa: investigación y pensamiento crítico, 9(1), 67-87. http://doi.org/10.17993/3cemp.2020.090141.67-87

MAPA (Ministerio de Agricultura Pesca y Alimentación). (2020). Vitivinicultura. https://www. mapa.gob.es/es/agricultura/temas/producciones-agricolas/vitivinicultura/default.aspx\#ancla6

OEMV. (2018). El número de bodegas en España sube hasta las 4.373. https://www.oemv.es/el-numero-debodegas-en-espana-sube-hasta-las-4-373

Oltra-Badenes, R., Gil-Gomez, H., Guerola-Navarro, V., y Vicedo, P. (2019). Is It Possible to Manage the Product Recovery Processes in an ERP? Analysis of Functional Needs. Sustainability, 11(16), 4380. https://doi.org/10.3390/su11164380

Tecnovino.com. (2018). Crece el número de bodegas en España en 2018, hasta 4.373. https:/ /www.tecnovino. com/crece-el-numero-de-bodegas-en-espana-en-2018-hasta-4-373/ 